\title{
Publisher Correction: Network inference from glycoproteomics data reveals new reactions in the IgG glycosylation pathway
}

\author{
Elisa Benedetti ${ }^{1}$, Maja Pučić-Baković ${ }^{2}$ Toma Keser (10 ${ }^{3}$, Annika Wahl ${ }^{4,5}$, Antti Hassinen ${ }^{6}$, Jeong-Yeh Yang7 , \\ Lin Liu7, Irena Trbojević-Akmačić², Genadij Razdorov (1) 2, Jerko Štambuk², Lucija Klarić2,8,9, Ivo Ugrina (1) 3,10,11, \\ Maurice H.J. Selman ${ }^{12}$, Manfred Wuhrer (10 ${ }^{12}$, Igor Rudan (10) ${ }^{8}$, Ozren Polasek ${ }^{13,14}$, Caroline Hayward (10 ${ }^{9}$, \\ Harald Grallert ${ }^{4,5,15}$, Konstantin Strauch ${ }^{16,17}$, Annette Peters ${ }^{5}$, Thomas Meitinger ${ }^{18}$, Christian Gieger ${ }^{4,5}$, \\ Marija Vilaj ${ }^{2}$, Geert-Jan Boons ${ }^{7,19}$, Kelley W. Moremen7, Tatiana Ovchinnikova ${ }^{20}$, Nicolai Bovin ${ }^{20}$, \\ Sakari Kellokumpu' ${ }^{6}$, Fabian J. Theis (10) 1,21, Gordan Lauc ${ }^{2,3}$ \& Jan Krumsiek (10) 1,15
}

Correction to: Nature Communications https://doi.org/10.1038/s41467-017-01525-0, published online 14 November 2017

The originally published version of this Article did not acknowledge Fabian J. Theis and Gordan Lauc as corresponding authors. Furthermore, the author Jan Krumsiek was incorrectly listed as being affiliated with institutions 1 and 14, and should have been affiliated with institutions 1 and 15. These errors have now been corrected in both the PDF and HTML versions of the manuscript.

Published online: 13 February 2018

\begin{abstract}
(c) (i)
Open Access This article is licensed under a Creative Commons Attribution 4.0 International License, which permits use, sharing, adaptation, distribution and reproduction in any medium or format, as long as you give appropriate credit to the original author(s) and the source, provide a link to the Creative Commons license, and indicate if changes were made. The images or other third party material in this article are included in the article's Creative Commons license, unless indicated otherwise in a credit line to the material. If material is not included in the article's Creative Commons license and your intended use is not permitted by statutory regulation or exceeds the permitted use, you will need to obtain permission directly from the copyright holder. To view a copy of this license, visit http://creativecommons.org/licenses/by/4.0/.
\end{abstract}

(C) The Author(s) 2018

\footnotetext{
${ }^{1}$ Institute of Computational Biology, Helmholtz Zentrum München-German Research Center for Environmental Health, 85764 Neuherberg, Germany.

${ }^{2}$ Genos Glycoscience Research Laboratory, 10000 Zagreb, Croatia. ${ }^{3}$ Faculty of Pharmacy and Biochemistry, University of Zagreb, 10000 Zagreb, Croatia.

${ }^{4}$ Institute of Epidemiology 2, Research Unit Molecular Epidemiology, Helmholtz Zentrum München-German Research Center for Environmental Health, 85764 Neuherberg, Germany. ${ }^{5}$ Institute of Epidemiology 2, Helmholtz Zentrum München-German Research Center for Environmental Health, 85764 Neuherberg, Germany. ${ }^{6}$ Faculty of Biochemistry and Molecular Medicine, University of Oulu, FI-90014 Oulu, Finland. ${ }^{7}$ Complex Carbohydrate Research Center, University of Georgia, Athens, GA 30602, USA. ${ }^{8}$ Usher Institute of Population Health Sciences and Informatics, University of Edinburgh, EH8 9 AG Edinburgh, UK. ${ }^{9}$ Medical Research Council Human Genetics Unit, Institute of Genetics and Molecular Medicine, University of Edinburgh, EH4 2XU Edinburgh, UK. ${ }^{10}$ Faculty of Science, University of Split, 21000 Split, Croatia. ${ }^{11}$ Intellomics Ltd., 10000 Zagreb, Croatia. ${ }^{12}$ Leiden University Medical Center, 2333 ZA Leiden, The Netherlands. ${ }^{13}$ University of Split School of Medicine, 21000 Split, Croatia. ${ }^{14}$ Gen-info Ltd., 10000 Zagreb, Croatia. ${ }^{15}$ German Center for Diabetes Research (DZD), 40225 Düsseldorf, Germany. ${ }^{16}$ Institute of Genetic Epidemiology, Helmholtz Zentrum München-German Research Center for Environmental Health, 85764 Neuherberg, Germany. ${ }^{17}$ Institute of Medical Informatics, Biometry and Epidemiology, Chair of Genetic Epidemiology, LudwigMaximilians Universität, 81577 Munich, Germany. ${ }^{18}$ Institute of Human Genetics, Helmholtz Zentrum München-German Research Center for Environmental Health, 85764 Neuherberg, Germany. ${ }^{19}$ Department of Chemical Biology and Drug Discovery, Utrecht Institute for Pharmaceutical Sciences, and Bijvoet Center for Biomolecular Research, Utrecht University, 3584 CG Utrecht, The Netherlands. ${ }^{20}$ Shemyakin and Ovchinnikov Institute of Bioorganic Chemistry, Russian Academy of Sciences, 117997 Moscow, Russia. ${ }^{21}$ Department of Mathematics, Technical University Munich, 85748 Garching bei München, Germany Correspondence and requests for materials should be addressed to F.J.T. (email: fabian.theis@helmholtz-muenchen.de) or to G.L. (email: glauc@genos.hr) or to J.K. (email: jan.krumsiek@helmholtz-muenchen.de)
} 